\title{
Targeting the epithelial growth factor receptor in head and neck tumours: current status and challenges
}

\author{
Loredana G. Marcu ${ }^{1,2 *}$ \\ ${ }^{1}$ Faculty of Science, University of Oradea, Romania \\ ${ }^{2}$ Division of Health Sciences, University of South Australia, SA 5005, Australia
}

(Received 27 November, 2017; accepted 28 January 2018)

\begin{abstract}
While locoregional control in head and neck cancer has improved over the last few decades because of advances in imaging and treatment technology, five-year survival rates are still poor due to tumour resistance to treatment, recurrence and metastatic spread. There are several known biological parameters that are strongly patient specific and guide tumour response, thus require treatment personalisation in order to further improve tumour control. Better tailored treatments such as molecular targeted therapies are therefore becoming widely implemented into clinics for a large range of malignancies. Head and neck cancers are no exceptions, as several molecular characteristics have been identified that can serve as possible therapeutic targets. The aim of this mini review is to revisit the role of epithelial growth factor receptor in head and neck carcinomas and to underline the current clinical challenges regarding treatment resistance.
\end{abstract}

Keywords: radiotherapy, EGFR, monoclonal antibodies, tyrosine kinase inhibitors

\section{INTRODUCTION}

Head and neck cancer is the sixth most common cancer worldwide and despite all efforts to improve treatment outcome, five-year survival rates are still poor due to tumour resistance to treatment, recurrence and metastatic spread (1). While chemoradiotherapy is the standard treatment for locally advanced head and neck cancer, one of its major disadvantages is the increased normal tissue toxicity that is often a dose-limiting factor (2). Whether cell cycle-specific or nonspecific, common chemotherapeutic drugs lack selectivity between healthy and malignant cells, raising the need for better defined targets (3).

Several molecular pathways and characteristics that provide valuable information concerning tumour progression, distant spread and response to treatment have been lately revealed in the context of head and neck cancer therapy. Figure 1 illustrates the main molecular targets that are currently in focus, such as the epithelial growth factor receptor (EGFR), the vascular endothelial growth factor (VEGF), insulin-like growth factor receptor (IGF-1R), proteasome inhibitors, Src kinase family and $\mathrm{PI} 3 \mathrm{~K} / \mathrm{AKT} / \mathrm{mTOR}$ pathway inhibitors.
Consequently, new therapeutic agents have been developed that target various signalling pathways with the aim of interfering with tumour progression $(4,5)$. However, the clinical results with EGFR inhibitors is controversial, and even in the best case the outcome is modest. Most often, this limitation is due to treatment resistance. Despite recent advances in this area, the mechanisms behind resistance to EGFR targeting agents in head and neck cancer are not fully elucidated.

The aim of this mini review is to revisit the role of epithelial growth factor receptor in head and neck carcinomas and to highlight the current clinical challenges concerning treatment resistance.

\section{THE EPITHELIAL GROWTH FACTOR RECEPTOR (EGFR) AND RADIOTHERAPY}

EGFR is a transmembrane tyrosine kinase receptor that regulates key transduction pathways in both healthy and malignant cells. The epidermal growth factor receptor belongs to the ErbB family of receptor tyrosine kinases, being part of four growth factor receptors: EGFR (or ErbB-1) and the human epidermal receptors HER-2 (c-ErbB-2), HER-3 (c-ErbB-3) and HER-4

${ }^{*}$ Corresponding author: Prof Loredana G. Marcu, PhD

Faculty of Science, University of Oradea, 410087, Romania

Tel: +40730669 502;

Email: loredana@marcunet.com 
(c-ErbB-4). Due to promotion of epidermal cell growth and regulation of cell proliferation, EGFR was shown to play a crucial role in head and neck cancer progression, angiogenesis and distant spread (6). Clinical studies have unanimously proven that overexpression of EGFR among HNC patients is highly common (about $90 \%$ of HNCs exhibit high levels of EGFR) and is also correlated with poor prognosis.

EFGR inhibitors have been designed as either monoclonal antibodies (mAb) or small molecules. While monoclonal antibodies target extracellular growth factors or specific antigens found on the cell surface, small molecules such as tyrosine kinase inhibitors (TKI) can reach the target by penetrating the cell membrane and interfere with its enzymatic activity. Anti-EGFR monoclonal antibodies prevent ligand binding by a competitive coupling to the extracellular area of EGFR, whereas small molecule inhibitors of the tyrosine kinase activity hinder intracellular downstream signalling (7). Of the EGFR inhibitors shown in Fig. 1, cetuximab is the only agent approved for the treatment of head and neck cancers. Nevertheless, predictive biomarkers of response to cetuximab are yet to be developed.

In order to modulate tumour response to radiation, Harari $e t$ al investigated the potential of EGFR blockade using mAbs (8). The pre-clinical experiments undertaken in squamous cell carcinoma lines showed that blockade of the EGFR pathway induces cell cycle arrest in the G1 and G2-M phases, while also decreasing the S-phase fraction. Given the fact that the $S$ phase is the most radioresistant phase of the cell cycle and, in contrast, G2-M the most radiosensitive, the build-up of cells in the more radiosensitive phases augments overall radiosensitivity induced by monoclonal antibodies. It was suggested that the modulation of radiation response by EGFR blockade is multifactorial, being driven by growth arrest, inhibition of DNA repair, increased apoptosis, and downregulation of angiogenic response.

While the interplay between radiation and EGFR could imply at least an additive effect of the two agents when administered concurrently, the complex tumour microenvironment interferes with the expected outcome and the clinical results are often suboptimal. Several factors have been identified that modulate the effect of EGFR inhibitors (9). Resistance mechanisms include but are not limited to: activation or upregulation of other ErbB family members, activation of parallel downstream signalling pathways, induction of epithelial to mesenchymal transitions (EMT) and interplay with tumour microenvironment.

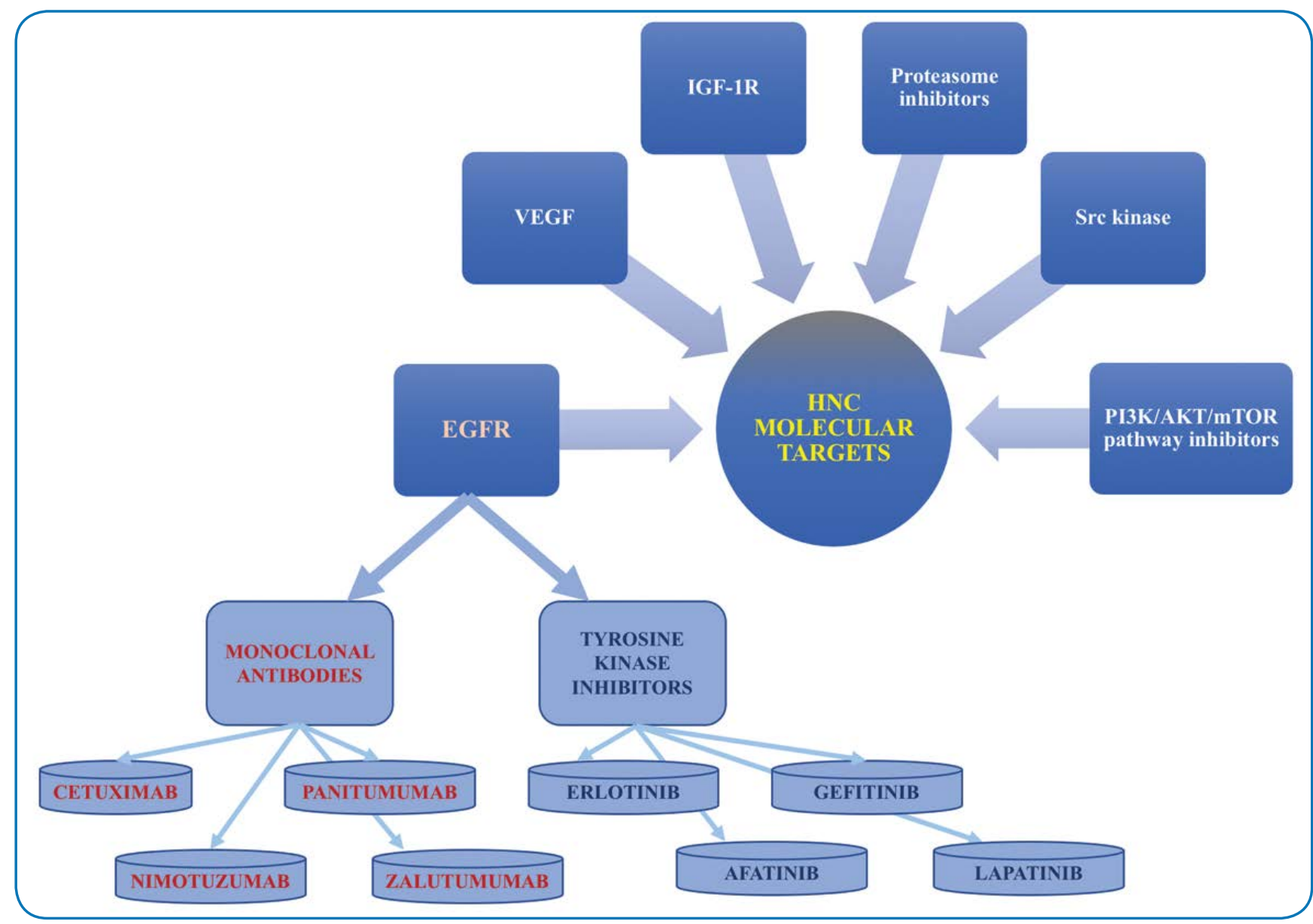

Fig. 1. Molecular targets for targeted therapy in head and neck cancer. The most representative EGFR inhibitors are presented in their respective categories (monoclonal antibodies and tyrosine kinase inhibitors). 


\section{CURRENT CHALLENGES: RESISTANCE TO ANTI-EGFR TREATMENT}

\section{Activation of parallel downstream signalling pathways}

Despite EGFR inhibition with targeting agents, there is experimental evidence towards parallel oncogenic signalling through $G$ protein-coupled receptors, showing that other downstream pathways remain activated during EGFR blockade (10). Identification and targeting of mediators of the cross talk between EGFR and G protein-coupled receptors could result into tumour growth inhibition and overall improvement of outcome.

It was hypothesised that the inhibition of the mammalian target of rapamycin complex (mTOR) could improve the effects of EGFR blockade in preclinical models, given the fact that mTOR is activated by both EGFR pathways as well as $G$ protein-coupled receptors (10). To prove the hypothesis, when erlotinib (an anti-EGFR small molecule) was combined with an mTOR inhibitor (OSI-027), a significant reduction in head and neck cancer (HNC) cell line proliferation was observed, the combined treatment demonstrating a synergistic inhibition of cell survival. Similar antitumour effects were obtained when cetuximab was combined with the mTOR inhibitor (10). These results are in correlation with the HNC xenograft studies reported by Wang et al (11), whereby cetuximab-resistant cells exhibited a substantial response to the combined mTOR/cetuximab therapy.

To date, co-targeting HNC with mTOR inhibitors to overcome drug resistance shows promising results, with some agents entering clinical trials (12). To gain more insight into the mechanisms behind treatment resistance, more research is warranted to investigate those downstream pathways that continue to be activated during EGFR blockade.

\section{Upregulation/activation of ErbB family members}

As shown above, EGFR is a member of the ErbB family. Activation of other members of the ErbB group can be a plausible cause for resistance to anti-EGFR therapy. In vitro studies of head and neck cancer cell lines treated with gefitinib (see Figure 1) showed resistance to this EGFR inhibitor through upregulation and activation of ErbB-2 and ErbB-3 receptors (13). It was revealed that cell lines that display resistance to EGFR therapy express high levels of ErbB-2 and ErbB-3, which could, therefore, become new targets. To test this, a humanized antibody targeting ErbB-2 was combined with gefitinib, combination that was effective in suppressing clonogenic survival in a dose-dependent manner.

Similar experiments were undertaken on cetuximab-resistant tumours showing that inhibition of ErbB-2 or disruption of ErbB-2/ErbB-3 heterodimerization can restore cetuximab sensitivity both in vitro and in vivo (14).

\section{Induction of epithelial to mesenchymal transitions}

Epithelial to mesenchymal transition (EMT) is a critical process in tissue homeostasis and plays a role in cancer progression. Cells that undergo EMT are characterised by the loss of cell-cell adhesion (E-cadherin, $\beta$-catenin) and the gain of mesenchymal markers (such as vimentin), leading to migratory functions (15). EMT processes in head and neck cancers are therefore responsible for the metastatic spread.

Overexpression of certain proteins that are involved in EMT have been associated with resistance to anti-EGFR treatment $(16,17)$. Pre-clinical studies in head and neck carcinomas have associated the overexpression of the transcription factor Snail with resistance to EGFR-targeting agents, such as erlotinib (16). This was mainly due to the loss of E-cadherin, implying that the maintenance of this tumour suppressor gene expression may support sensitivity to targeted therapy.

While investigating the link between EMT and resistance to EGFR-targeting therapy, Haddad et al showed that drugresistant cell lines expressed low levels of E-cadherin and high levels of the E-cadherin repressor deltaEF1 mesenchymal marker, having high migratory potential (17). When deltaEF1 expression was knocked down in the erlotinibresistant cell lines, this resulted in increased expression of E-cadherin and increased sensitivity to anti-EGFR therapy, thus demonstrating a cause-effect relationship between deltaEF1 expression and resistance to erlotinib.

Further investigations are warranted to develop agents that are capable of reversing EMT in head and neck cancers in order to reinstate sensitivity to EGFR inhibitors.

\section{EGFR and the hypoxic tumour environment}

Tumour hypoxia is a strong negative prognostic factor for treatment outcome and is the leading cause of resistance to radiotherapy. Hypoxia in head and neck cancer patients is very common, with about $75 \%$ of patients presenting with this condition, thus great efforts are invested into detection, quantification and management of hypoxia (18). Moreover, hypoxia is known to be a promoter of angiogenesis, and of distant metastases, driven by the hypoxia-inducible factor- $1 \alpha(\mathrm{HIF}-1 \alpha)$ that regulates several processes along the metastatic pathway.

The hypoxic tumour environment was hypothesised to be involved in drug resistance against EGFR inhibitors. Given that colocalization of hypoxia and EGFR markers are correlated with poor outcome, a recent study employing cetuximab and erlotinib, the most representative anti-EGFR agents in their corresponding groups, established a dose-dependent growth inhibition under normal and hypoxic $\left(0 \% \mathrm{O}_{2}, 5 \% \mathrm{CO}_{2}, 95 \% \mathrm{~N}_{2}\right)$ conditions alike, in three different HNC cell lines (19). Interestingly enough, the study could not demonstrate any hypoxia-induced resistance, thus no predictive biomarkers could be identified for drug resistance under hypoxic conditions. Since 
this study was undertaken on cell lines, no other tumour microenvironmental factors beside hypoxia were considered.

To support the above observation, several current pre-clinical studies investigating the correlation between EGFR and hypoxia were able to demonstrate downregulation of EGFR in hypoxic tumour areas (20). Considering that EGFR-induced cell proliferation is strongly dependent upon the oxygenation status, meaning that the lack of oxygen limits the expression of EGFR, this result is highly plausible. While hypoxia might not be the only factor within the tumour microenvironment responsible for EGFR downregulation, it surely plays an important role in the process. Therefore, in patients treated with combined anti-EGFR radiotherapy, downregulation of EGFR could lead to treatment resistance.

\section{CONCLUSIONS AND PERSPECTIVES}

Advanced head and neck cancers are aggressive tumours that require multidisciplinary approach for their clinical management. While combined chemo-radiotherapy is the standard of care, the poor long-term survival entails therapies that are better targeted. Knowing that the

\section{REFERENCES}

1. Siegel RL, Miller KD, Jemal A, Cancer statistics, 2016, CA: A Cancer Journal for Clinicians, 66, 7-30 (2016).

2. Marcu LG, Improving therapeutic ratio in head and neck cancer with adjuvant and cisplatin-based treatments,

BioMed Research International, 817279 (2013).

3. Allen B, Bezak E, Marcu L, Quo vadis, radiotherapy? Technological advances and the rising problems in cancer management, Biomed Research International, 749203 (2013).

4. Bozec A, Peyrade F, Milano G, Molecular targeted therapies in the management of head and neck squamous cell carcinoma: recent developments and perspectives, Anticancer Agents Med. Chem., 13, 389-402 (2013).

5. Dorsey K, Agulnik M, Promising new molecular targeted therapies in head and neck cancer, Drugs, 73, 315-325 (2013).

6. Mendelsohn J, Baselga J, The EGF receptor family as targets for cancer therapy, Oncogene, 19, 6550-6565 (2000).

7. Ciardello F, Tortora G, $A$ novel approach in the treatment of cancer: Targeting the epidermal growth factor receptor,

Clin. Cancer Res., 7, 2958-2970 (2001).

8. Harari PM, Huang SM, Head and neck cancer as a clinical model for molecular targeting of therapy: Combining EGFR blockade with radiation, Int. J. Radiat. Oncol. Biol. Phys., 49, 427-433 (2001).

9. Campbell NP, Hensing TA, Bhayani MK, Shaikh AY, Brockstein $\mathrm{BE}$, Targeting pathways mediating resistance to anti-EGFR therapy in squamous cell carcinoma of the head and neck, Expert Rev. Anticancer Ther., 16, 847-858 (2016).

10. Cassell A, Freilino ML, Lee J, Barr S, Wang L, Panahandeh MC, et al, Targeting TORC1/2 Enhances Sensitivity to EGFR Inhibitors in Head and Neck Cancer Preclinical Models,

Neoplasia, 14, 1005-1014 (2012).

11. Wang Z, Martin D, Molinolo AA, Patel V, Iglesias-Bartolome R, Degese MS, et al, mTOR co-targeting in cetuximab resistance in head and neck cancers harboring PIK3CA and RAS mutations,

J. Natl. Cancer Inst., 106, pii: dju215 (2014).

12. Wang Z, Valera JC, Zhao X, Chen Q, Silvio Gutkind J, $m$ TOR epidermal growth factor receptor is overexpressed in this patient group, a logical solution to this problem would be the development of EFGR targeting agents, task that was fulfilled via monoclonal antibodies and small molecule tyrosine kinase inhibitors. To date, one such monoclonal antibody, cetuximab, is the only FDA-approved agent used in clinical settings, though with varied success. This is mainly due to treatment resistance that occurs due to several factors including: activation/ upregulation of other ErbB family members, activation of parallel downstream signalling pathways, induction of epithelial to mesenchymal transitions and the complex interplay with tumour microenvironment.

In order to overcome resistance to the currently tested EGFR inhibitors, the following are the perspectives: (1) the clinical implementation of new anti-EGFR agents that are able to target multiple members of the ErbB family (afatinib, lapatinib, see Fig. 1); (2) the combined administration of EGFR inhibitors with agents that target parallel downstream signalling pathways and have different mechanisms of action; (3) more insights into the role of tumour microenvironment in regulating EGFR expression; (4) identification of biomarkers for response to EGFR inhibitors.

co-targeting strategies for head and neck cancer therapy,

Cancer Metastasis Rev., 36, 491-502 (2017).

13. Erjala K, Sundvall M, Junttila TT, Zhang N, Savisalo M, Mali P, et al, Signaling via ErbB2 and ErbB3 associates with resistance and epidermal growth factor receptor (EGFR) amplification with sensitivity to EGFR inhibitor gefitinib in head and neck squamous cell carcinoma cells, Clin. Cancer Res., 12, 4103-4111 (2006).

14. Yonesaka K, Zejnullahu K, Okamoto I, Satoh T, Cappuzzo F, Souglakos J, et al, Activation of ERBB2 signaling causes resistance to the EGFR-directed therapeutic antibody cetuximab,

Sci. Transl. Med., 3, 99ra86 (2011).

15. Thiery JP, Acloque H, Huang RY, Nieto MA, Epithelial-mesenchymal transitions in development and disease, Cell, 139, 871-90 (2009).

16. Dennis M, Wang G, Luo J, Lin Y, Dohadwala M, Abemayor E, et al, Snail controls the mesenchymal phenotype and drives erlotinib resistance in oral epithelial and head and neck squamous cell carcinoma cells,

Otolaryngol. Head Neck Surg., 147, 726-732 (2012).

17. Haddad Y, Choi W, McConkey DJ, The transcriptional repressor Delta EF1 controls the EMT phenotype and resistance to the EGFR inhibitor erlotinib in human head \& neck squamous cell carcinoma lines, Clin. Cancer Res., 15, 532-542 (2009).

18. Chirla R, Marcu L, PET-based quantification of statistical properties of hypoxic tumor subvolumes in head and neck cancer, Phys. Med., 32, 23-35 (2016).

19. Boeckx C, Van den Bossche J, De Pauw I, Peeters M, Lardon F, Baay M, et al, The hypoxic tumor microenvironment and drug resistance against EGFR inhibitors: preclinical study in cetuximab-sensitive head and neck squamous cell carcinoma cell lines, BMC Res. Notes, 8, 203 (2015).

20. Mayer A, Zahnreich S, Brieger J, Vaupel P, Schmidberger H, Downregulation of EGFR in hypoxic, diffusion-limited areas of squamous cell carcinomas of the head and neck, Br. J. Cancer, 115, 1351-1358 (2016). 